\title{
Evolution of Leucyl-tRNA Synthetase Through Eukaryotic Speciation
}

\author{
Katelyn E. Unvert', Frank A. Kovacs, Chi Zhang, Rachel A. Hellmann-Whitaker and Katelin N. Arndt** \\ ${ }^{a}$ Department of Biology, University of Nebraska, Kearney \\ ${ }^{b}$ School of Biological Sciences, University of Nebraska, Lincoln \\ 'Department of Chemistry and Biochemistry, University of Arizona \\ Students:arndtkn@lopers.unk.edu*,unvertke@lopers.unk.edu \\ Contributing authors: zhang.chi@unl.edu,kovacsfa@unk.edu \\ Mentor: hellmann@email.arizona.edu
}

\begin{abstract}
Aminoacyl-tRNA synthetases (aaRSs) are part of the cellular translation machinery and as such, they are essential enzymes for every known cell. Due to their ubiquitous nature, their evolutionary history has been intensely researched to better understand the origins of life on a molecular level. Herein, we examine the evolutionary relatedness of leucyl-tRNA synthetases (LeuRS) from each major eukaryotic branch through the speciation process. This research effort was centered on amino acid sequence data as well as generating homology protein models for each LeuRS enzyme. Comparative analysis of this sequence and structural data for LeuRS amongst eukaryotes has indicated a high level of conservation within the active sites of these enzymes. Phylogenetic analysis confirmed this high degree of conservation as well as established evolutionary relatedness between these LeuRS enzymes. Based on this data, vertical gene transfer propagated LeuRS throughout the eukaryotic domain. Horizontal gene transfer and domain acquisition events were not observed within the eukaryotic organisms studied. Our data also highlighted LeuRS adaptation through the speciation process due to slight variability of scaffolding residues outside of the active site regions. We hypothesize that this variability may be due to mechanistic differences amongst LeuRS enzymes that have assumed nontranslational functionality through the evolutionary process.
\end{abstract}

\section{KEYWORDS}

tRNA Synthetase; Leucyl-tRNA Synthetase; Eukaryotic Evolution; LeuRS Conservation; Vertical Gene Transfer; Horizontal Gene Transfer; Convergent Evolution; Primordial Enzymes

\section{INTRODUCTION}

Aminoacyl-tRNA synthetases (aaRSs) are the evolutionary link between an ancient RNA-based world and the contemporary amino acid-driven world. ${ }^{1}$ Hypotheses developed from substantiated data has determined that aaRSs are an ancient family of enzymes that displayed greater fitness for aminoacylation catalysis, thus displacing ribozymes from this catalytic niche. ${ }^{1-2}$ As such, aaRSs chemically link nucleic acids and amino acids through the aminoacylation reaction, which plays a central role in translation. ${ }^{3}$ The aminoacylation reaction consists of aaRSs binding to their cognate amino acid as well as tRNA to generate a hybrid aminoacylated tRNA structure. ${ }^{4}$ Thus, aaRSs are ubiquitous throughout nature and as such their evolutionary origins have been the subject of numerous research studies., 5-9

It is thought that aaRSs evolved to better adapt an existing genetic code to a changing biological environment, in which the kinetic efficiency of the aminoacylation reaction needed to improve to meet the needs of a developing primordial cell or cellular community. ${ }^{7-8}$ The intense evolutionary pressure that existed in the early stages of the emergence of life resulted in evolutionary convergence within the aaRS family of enzymes. ${ }^{1}$ This family of enzymes was thus subdivided into class I and class II aaRSs, with the result: one aaRS per amino acid. ${ }^{10-11}$ Interestingly, class I and class II aaRSs are structurally dissimilar, however, this family of enzymes almost evenly divided with approximately 10 aaRSs in each subgroup. ${ }^{10-13}$ This suggests that a level of evolutionary coordination existed between these two classes of aaRSs.

Given that the family of aaRSs is comprised of approximately 20 enzymes and that there is high catalytic fidelity displayed by each enzyme, the evolutionary path for each class of aaRS necessitated structural divergence from two separate ancient aaRS primordial 
enzymes. ${ }^{5}$ Divergence and propagation of each aaRS subclass may have been precipitated through horizontal gene transfer (HGT), gene duplication, and domain acquisition events. ${ }^{7-9}$ Evidence of HGT and domain acquisition events within the family of aaRSs have been used to explain the complicated phylogeny of these enzymes, since they do not seem to adhere to the canonical organismal phylogeny proposed by Carl Woese. 5, 7, 14-16 Thus, the molecular evolution of aaRSs is a complex story, in which the details are continuing to unfold.

Class I aaRSs are multi-domain enzymes, with the main domain harboring the aminoacylation active site, with the core catalytic site being comprised of a Rossmann nucleotide binding fold. ${ }^{17-18}$ The Rossmann fold binds to cognate amino acid and tRNA as well as ATP to catalyze the aminoacylation reaction. ${ }^{10}$ Several of the class I aaRSs have a secondary editing domain that is an insertion into the Rossmann fold and is thought to be a later evolutionary addition to these enzymes. ${ }^{16,19}$ In contrast to class I aaRSs, the class II aaRS active site is comprised of antiparallel $\beta$-strands, which are flanked by $\alpha$-helices. ${ }^{20}$

The leucyl-tRNA synthetase (LeuRS) enzyme is a class I aaRS that is thought to have diverged from a common ancestral aaRS that also gave rise to isoleucyl-tRNA synthetase (IleRS) and valyl-tRNA synthetase (ValRS). ${ }^{16},{ }^{21}$ This divergence event is thought to have occurred before the time of the last universal common ancestor (LUCA). ${ }^{9}$ Originally, it was thought that the LeuRS molecular phylogeny was one of the only examples of aaRSs that followed Carl Woese's Archaea-Bacteria-Eukaryota trichotomy. ${ }^{14}$ However, other research evidence suggests that the LeuRS evolutionary history may have been subject to HGT events, as is the case for other aaRSs. ${ }^{7-9}$ These HGT events complicate the molecular phylogeny of LeuRS, but may help explain its ubiquitous nature as well as its biochemical conservation through the speciation process of all three domains of life.

In this study, we analyzed the sequence and structural features of LeuRS enzymes through the eukaryotic lineage to establish evolutionary relatedness. Though we focused our research efforts on LeuRS divergence through the speciation of eukaryotes, we started our comparative analysis with a bacterial example of LeuRS since it is thought that the Archeae and Eukaryota branches split from the bacterial branch. ${ }^{22}$ Highlighting LeuRS evolution through eukaryotic speciation, starting from a bacterial example, shed light on LeuRS propagation throughout the eukaryotic domain of life. Additionally, these studies demonstrate the possibility of pharmacological cross-reactivity potential amongst this group of LeuRS enzymes.

\section{METHODS AND PROCEDURES}

Sequence Comparisons

Multiple sequence alignments were conducted with amino acid sequences for LeuRS enzymes from the following organisms with the corresponding National Center for Biotechnology Information (NCBI) protein identification numbers: Escherichia coli (BAA35289.1), Saccharomyces cerevisiae (EDN60985.1), Arabidopsis thaliana (NP_172433.2), Draosophila melanogaster (AGV77169.1), Danio rerio (XP_698279.6), Xenopus laevis (NP_001087393.1), Anolis carolinensis (XP_003224146.1), Taeniopygia guttata (XP_002189937.1), Mus musculus (NP_598898.2), and Homo sapiens (NP_064502.9). Retrieval of these protein sequences was achieved with the Basic Local Alignment Search Tool (BLAST). ${ }^{23}$ These sequences were then subjected to various multiple sequence alignment analyses using Clustal Omega and PRALINE. ${ }^{24-25}$ The PRALINE sequence alignment results were used to determine relative conservation between LeuRS enzymes, which was in turn used in determining LeuRS structural comparisons.

An additional multiple sequence alignment was conducted using tRNA Leu sequence data from the following sources: Escherichia coli tRNA ${ }^{\text {Leu }}$ sequences with the anticodons: CAA, CAG, GAG, TAA and TAG; Saccharomyces cerevisiae tRNA ${ }^{\text {Leu }}$ sequences with the anticodons: TAA, CAA and TAG; Homo sapiens tRNALeu sequences with the anticodons: TAA, AAG and TAG. These tRNALeu sequences were retrieved from the tRNA Genomic Database. ${ }^{26}$ The subsequent multiple sequence alignment analysis was conducted using the T-Coffee alignment tool. ${ }^{27}$

Homology Protein Modeling and Structural Comparisons

To generate the LeuRS homology models, in silico protein structure prediction techniques were utilized. The development and refinement of these LeuRS computational models, including fold recognition methodologies, were conducted using SPARKX and I-TASSER software..$^{28-30}$

The I-TASSER platform is commonly used to generate 3D models of a target protein sequence. It utilizes a composite method that involves amino acid threading, which is guided by sequence alignment and secondary structure prediction paired with various 
structural refinement techniques. Each final homology model is evaluated via a confidence score (C-score), which is based on the threading template alignment quality as well as the convergence of structures coming from the assembly simulations. The Cscores are usually in the range of -5 to 2 with values above -1.5 considered to be significantly more reliable. ${ }^{30}$ Additionally, the models are evaluated by a TM-score, which measures structure similarity of the best threading alignment with the average of all the alignments generated. Models with TM-scores above 0.5 are considered topologically reliable. ${ }^{30}$ Please refer to Table 1 for a complete listing of these reliability scores.

\begin{tabular}{|l|l|l|}
\hline \multicolumn{1}{|c|}{ Organism } & C-score & \multicolumn{1}{c|}{ TM-score } \\
\hline Arabidopsis thaliana & 0.44 & $0.77+/-0.1$ \\
\hline Draosophila melanogaster & -1.18 & $0.57+/-0.15$ \\
\hline Xenopus laevis & -1.51 & $0.53+/-0.15$ \\
\hline Homo sapiens & -1.57 & $0.52+/-0.15$ \\
\hline Mus musculus & -1.55 & $0.52+/-0.15$ \\
\hline Saccharomyces cerevisiae & 0.56 & $0.79+/-0.09$ \\
\hline Taeniopygia guttata & -1.29 & $0.55+/-0.15$ \\
\hline Danio rerio & -1.11 & $0.58+/-0.14$ \\
\hline Anolis carolinensis & -1.33 & $0.52+/-0.14$ \\
\hline
\end{tabular}

Table 1. I-TASSER Reliability Scores for all Homology models.

Once the protein homology models were generated, various structural comparisons were made through their superimposition. This was accomplished through the Chimera software suite. ${ }^{31-33}$ Additional structural manipulations, coloring changes, protein fragmentation as well as rotations were also done using the Chimera software suite.

\section{Phylogenetic Tree Development}

Determination of LeuRS amino acid sequence conservation as well as evolutionary relatedness was confirmed with the development of a phylogenetic tree. This phylogenetic tree was generated using the following LeuRS amino acid sequences: Escherichia coli (BAA35289.1), Saccharomyces cerevisiae (EDN60985.1), Arabidopsis thaliana (NP_172433.2), Draosophila melanogaster (AGV77169.1), Danio rerio (XP_698279.6), Xenopus laevis (NP_001087393.1), Anolis carolinensis (XP_003224146.1), Taeniopygia guttata (XP_002189937.1), Mus musculus (NP_598898.2), and Homo sapiens (NP_064502.9). The phyloT: a tree generator software program was utilized to compile this sequence information into a final phylogenic tree. ${ }^{34-35}$

\section{RESULTS}

The process of modern translation of the genetic code is facilitated by an ancient family of enzymes called aminoacyl-tRNA synthetases (aaRSs). ${ }^{36}$ In vitro evolution experiments of ribozymes with aminoacylation activity, suggests that as the ancient RNAbased world evolved, aaRSs may have been one of the first enzymes to develop and displace this ribozyme catalytic niche. ${ }^{1-2}$ As such, it has been hypothesized that the genetic code pre-dates the existence of aaRSs, and due to evolutionary pressure to improve the catalytic efficiency of the aminoacylation reaction, amino acid-based primordial aaRSs evolved. ${ }^{7}$ These ancestral aaRSs structurally diverged into a family of 20 aaRS enzymes: one for each canonical amino acid. ${ }^{7}$ This structural divergence is largely attributed to horizontal gene transfer (HGT) and domain acquisition events that predate the time of the last universal common ancestor (LUCA). ${ }^{7} 9$ Further structural divergence of aaRSs occurred as the speciation process gave rise to the three domains of life. ${ }^{7}$ Interestingly, aaRS domain arrangement and their basic catalytic features have remained relatively unchanged since the time of LUCA. ${ }^{9}$ The ancient origins of a particular aaRS, leucyl-tRNA synthetase (LeuRS), has been well characterized. ${ }^{8}$ This research effort focuses on the structural divergence of LeuRS through the evolution of the eukaryotic lineage starting from bacteria.

The LeuRS enzyme consists of two main catalytic centers, housed within the aminoacylation domain and the connective polypeptide 1 (CP1) domain (Fig. 1). ${ }^{19}, 37$ The aminoacylation domain active site binds to leucine, ATP and cognate tRNALeu to facilitate the aminoacylation reaction. ${ }^{3,} 38$ The CP1 domain active site hydrolyzes misaminoacylated products that are generated by the aminoacylation reaction. ${ }^{39}$ The LeuRS aminoacylation domain is hypothesized to be the most ancient proteinaceous functional unit of LeuRS, evolving from a common ancestor aaRS that structurally diverged into LeuRS, IleRS and ValRS. ${ }^{16,21}$ Using 
BLAST, Clustal Omega and PRALINE multiple sequence alignment tools, we generated numerous sequence alignments of the catalytic core of the LeuRS aminoacylation domain from Escherichia coli (E. coli), Saccharomyces cerevisiae (S. cerevisiae), Arabidopsis thaliana (A. thaliana), Draosophila melanogaster (D. melanogaster), Danio rerio (D. rerio), Xenopus laevis (X. laevis), Anolis carolinensis (A. carolinensis), Taeniopygia guttata (T. guttata), Mus musculus (M. musculus) and Homo sapiens (H. sapiens)(Fig. 2A). This sequence alignment focuses on the signature sequences HIGH and KMSKS from the Rossmann nucleotide binding motif, which is the catalytic core of the LeuRS aminoacylation domain. ${ }^{3}$ The sequence alignment of all the organisms in Figure $2 \mathbf{A}$ indicates that there is considerable conservation within these signature sequences, underscoring their essential function as well as their ancient nature. The PRALINE sequence alignment tool uses a scoring scheme based on conservation, with 0 being the least conserved and 10 being the most conserved. This numeric gradient is paired with a color gradient where 0 is dark blue and 10 is red. As seen in Figure 2A, all the positions within the HIGH and KMSKS signature sequences have conservation values of 6 or greater, excluding E. coli LeuRS. Due to E. coli LeuRS being significantly smaller than the eukaryotic LeuRS enzymes and algorithmic limitations of this sequence alignment tool, the E. coli KMSKS region was not included in this sequence alignment. However, the E. coli LeuRS KMSKS region can be visualized in Figure 2B. Figure $2 \mathbf{B}$ highlights the extreme conservation of the aminoacylation active site within this diverse group of organisms, in which LeuRS homology protein structures were predicted and the structure of the HIGH and KMSKS regions from each organism were superimposed. Based on these results, and given the fact that these organisms span approximately 3.4 billion years of evolutionary time, it is remarkable that these signature sequences and their corresponding structures have remained largely unchanged. ${ }^{40}$ This supports the hypothesis that LeuRSs' catalytic core domain originated prior to the time of LUCA and remained largely unchanged over billions of years. ${ }^{8}$ Thus, vertical gene transfer events predominated the propagation of LeuRS throughout the eukaryotic domain.

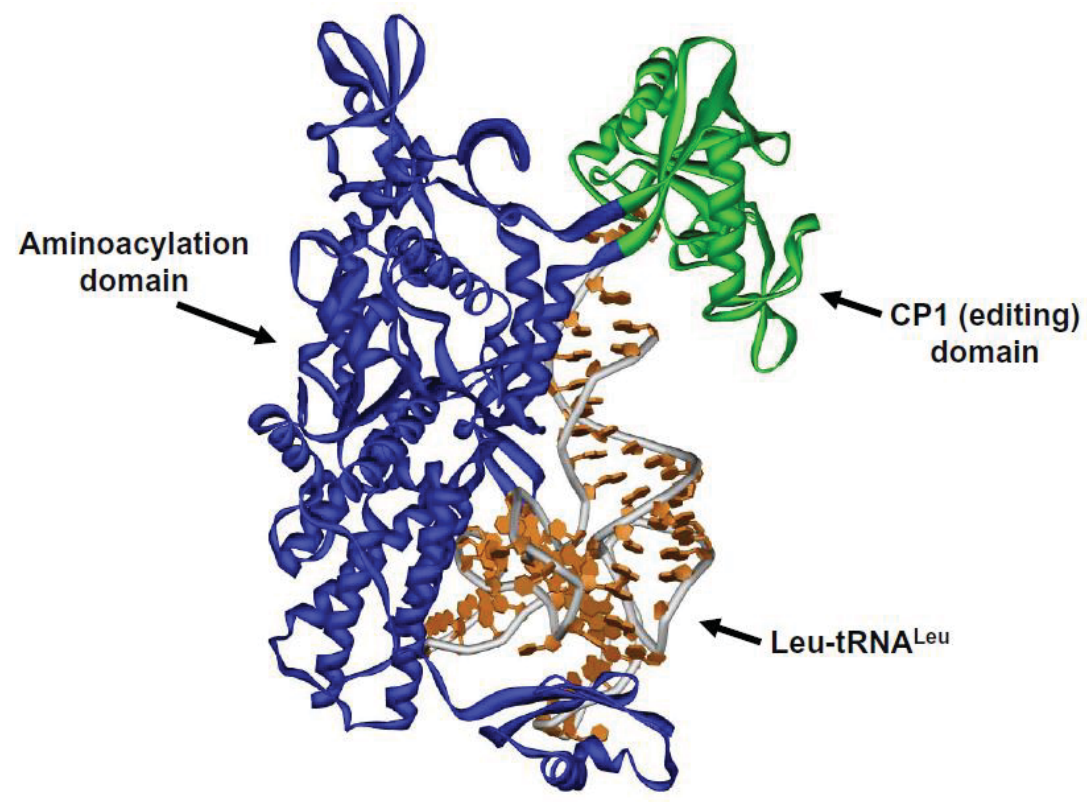

Figure $1 .{ }^{37} \mathrm{X}$-ray crystallography structure of E. coli LeuRS. The E. coli LeuRS crystal structure while in the editing conformation. The aminoacylation domain is blue and the CP1 editing domain is green. The bound Leu-tRNA ${ }^{\text {Leu }}$ is highlighted in orange. 


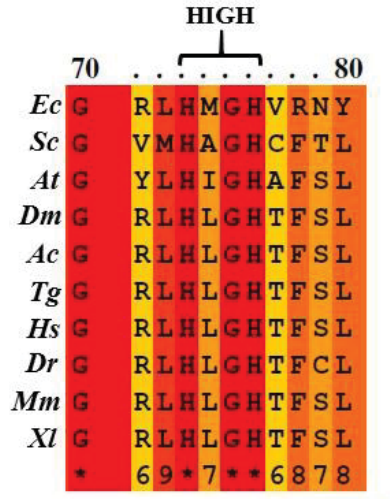

Unconserved 0123456789 IIIConserved

Figure 2A.

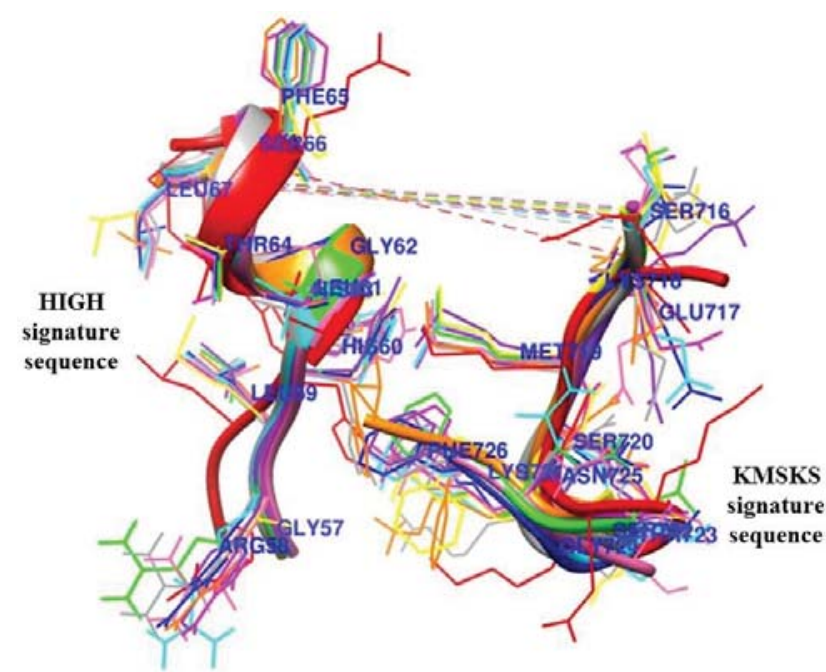

Figure 2B.

Figure 2. Multiple sequence alignment (2A.) and superposition (2B.) of the Aminoacylation active site "HIGH" and "KMSKS" signature sequence regions. 2A. ${ }^{3}$ Brackets above the multiple sequence alignment highlight the "HIGH" and "KMSKS" signature sequences, which are universal sequences found within the LeuRS active site. The signature sequences bind and stabilize ATP and the adenylate intermediate.

2B. ${ }^{37}$ The E. coli LeuRS X-ray crystallography structure is red. The E. coli LeuRS crystal structure is overlaid with the following computationally generated LeuRS homology models. Organism abbreviations and color labels are as follows: Eschericbia coli (Ec), Saccharomyces cerevisiae (Sc) (yellow), A rabidopsis thaliana (At) (purple), Draosophila melanogaster (Dm) (green), Danio rerio (Dr) (orange), Xenopus laevis (Xl) (blue), Anolis carolinensis (Ac) (magenta), Taeniopygia guttata (Tg) (cyan), Mus musculus $(\mathrm{Mm})$ (gray) and Homo sapiens (Hs) (pink).

Additional support for this hypothesis is displayed in the superimposed structures of the entire catalytic core docking site for ATP, leucine and the 3' acceptor stem end of tRNA Leu from E. coli and S. cerevisiae (Fig. 3), which indicates a high level of preservation through eukaryotic evolution. The E. coli LeuRS structure is red and the S. cerevisiae LeuRS structure is yellow. Given that these organisms are thought to be separated by 1.5 billion years of evolutionary time and are structurally dissimilar in many other ways (refer to Fig. 7), it is remarkable the that active sites of these two enzymes are extremely similar. ${ }^{40}$ This result provides evidence that the LeuRS active site originated from a common ancestral aaRS and has virtually remained unchanged. Additionally, these results support the assertion that the root of the three domains of life be placed along the bacterial branch with the Archaea and Eukaryota branches being clustered closely together. ${ }^{22,41}$

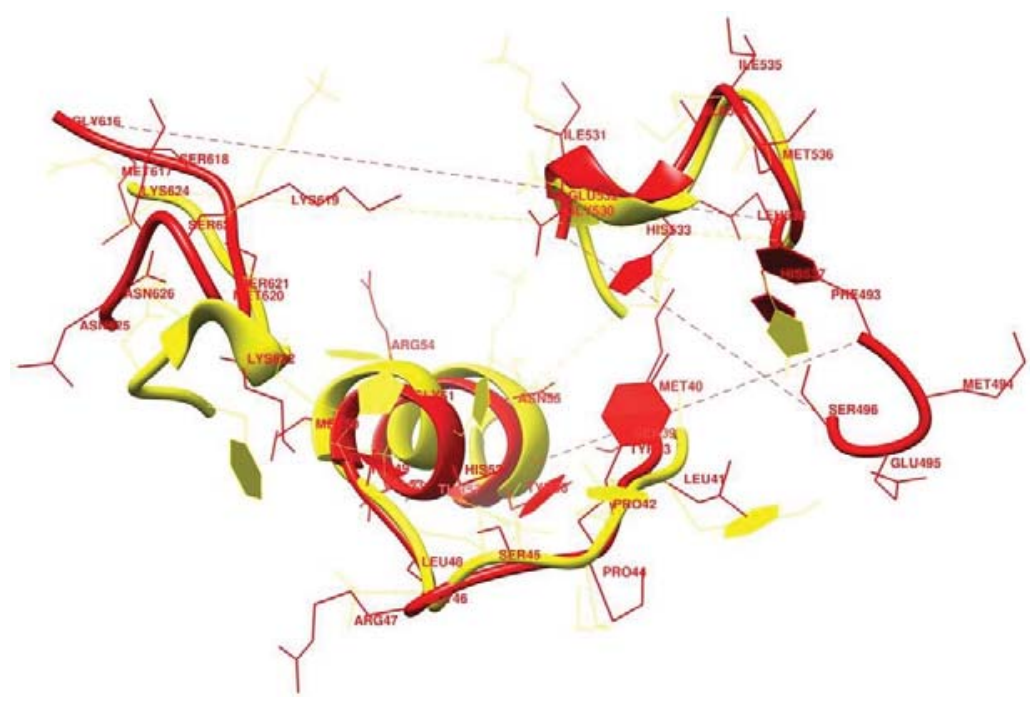

Figure 3. ${ }^{37}$ Aminoacylation active site superimposition of E. coli and S. cerevisiae. Superimposition of the entire aminoacylation active site. The E. coli LeuRS X-ray crystallography structure is red and the $S$. cerevisiae LeuRS homology model is yellow. 


\begin{tabular}{|c|c|c|c|c|c|}
\hline Escherichia_col & N I A Y MKNQLK & MLGFGYDWSR & ELATCT- PE & YYRWEQKFFT & E I Y K K G L VYK \\
\hline ccharomyces_c & FP P L E SDCT & SLGARIDWRR & SFVTTDANPY & YDAFIRWQMN & KLKAAGKIKF \\
\hline rabidopsis_tha & FP L AVEDLR & AYGLGCDWRR & SFVTTDVNPF & FDAFVRWQMR & KIKSMGKIVK \\
\hline Drosophila_mela & FP P AVQDLK & RIGVHVDWRR & TFITTDANPY & FDSFVRWQFN & HLKERGKIMY \\
\hline Anolis_caroline & FP P L A I QD L K & SMGLKIDWRR & SFVTTDVNPY & YDSFVRWQFL & TIRERNKIKF \\
\hline Taeniopygia_gut & FP P L A QDLK & SMGLKVDWRR & SF I T TNVNPY & YDSFVRWQFL & TLKERNKIKF \\
\hline Homo_sapiens & FP D I I QDLK & RMGLKVDWRR & SFITTDVNPY & YDSFVRWQFL & TIRERNKIKF \\
\hline Danio_rerio & FPPLAVEDLK & RMGLKVDWRR & SFITTDVNPF & YDSFVRWQFI & NLKERKKIKF \\
\hline Mus_musculus & FP L AVQDLK & TIGLKVDWRR & SFITTDVNPY & YDSFVRWQFL & TLRERNKIKF \\
\hline Xenopus_laevis & F P P LAVEDLK & SMGLKVDWRR & SF I T TDVNPF & YDSFVKWQFV & K L KERNKIKF \\
\hline \multirow[t]{2}{*}{ Consistency } & 8888766888 & $56 \star 757 \star \star 8 \star$ & $888 \star 8768$ * & 8878888874 & 758966 \\
\hline & $\ldots \ldots \ldots 260$ & $\ldots \ldots 270$ & 280 & $\ldots \ldots \ldots 290$ & \\
\hline Esc & 1 KTSAVNWCPN & DQTVLANEQV & IDGCCWRCDT & KVERKEI PQW & FIKITAYADE \\
\hline s_c & CGERYTIYSEK & $D G Q A C M D H D R$ & QSG- ----- & $--\mathrm{EGVT}-\mathrm{PQE}$ & YIGVKIEALE \\
\hline Arabidopsis_tha & DRRYTIFSPL & $D G Q P C A D H D R$ & A T G- - - - - & $--E G V Q-P Q E$ & YTLIKME- - \\
\hline Dros & a GKRYTIYSPK & DGQPCMDHDR & SSG-- - - - & $--E G V G-P Q E$ & YTLI KMK- - \\
\hline Anoli & GKRYTIYSPK & $D G Q P C M D H D R$ & QT G - & $--E G V G-P Q E$ & YTLIKMK-- - \\
\hline opygia_gut & GKRYTIYSPK & DGQPCMDHDR & $\mathrm{QTG}-\cdots-\cdots$ & $--E G V G-P Q E$ & YTL I KMK- - - \\
\hline Homo_sapiens & GKRYTIYSPK & $D G Q P C M D H D R$ & QTG-- - - - & $--E G V G-P Q E$ & YTLLKLK--- \\
\hline Danio_r & GKRYTIYSPK & DGQPCMDHDR & QTG-- - - - - & $--\mathrm{EGVG}-\mathrm{PQE}$ & YTL I KMK--- \\
\hline Mus_musculus & GKRYTIYSPK & $D G Q P C M D H D R$ & QTG-- - - - - & $--E G V G-P Q E$ & YTLVKLK--- \\
\hline Xenopus_laevis & GKRYTIYSPR & $D G Q P C M D H D R$ & $\mathrm{QTG}-\ldots-\ldots$ & $--\mathrm{EGVG}-\mathrm{PQE}$ & YTL I KMK-- - \\
\hline \multirow[t]{2}{*}{ Consistency } & 7788888886 & $\star 887879888$ & $67 \star 0000000$ & $00 * 8850 * \star 8$ & 9769877000 \\
\hline & 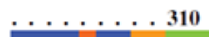 & 320 & 33 & $\ldots \ldots$ & $\ldots .350$ \\
\hline $\operatorname{col}$ & $1 \mathrm{LLNDLDKLDH}$ & WPDTVKTMQR & NW I GRSEGVE & ITENVNDYDN & TLTVYTTRPD \\
\hline Sacc & CFADDA--AKI & IDSSSDLDKS & KKF------ & - - - - - - - & YFVAATLRPE \\
\hline tha & $a----V--V K P$ & FPLKLGPLEG & KRV ------- & --------- & FLAAATLRPE \\
\hline Drosor & $a----V--L E-$ & VPKALSSI-K & QP I - - - - - & 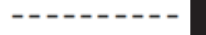 & FMVA ATLRPE \\
\hline Anol & $----V--V E P$ & Y PVKLSGLKG & KN I - - - - - & ---------- & FLVAATLRPE \\
\hline Taen & $t----V--L D P$ & YPAKLSGLRG & KN I------ & --------- & FLVAATLRPE \\
\hline Hom & $----V--L E P$ & YPSKLSGLKG & KNI---- - & 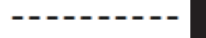 & FLVAATLRPE \\
\hline Dan & $----V--V E P$ & YPTKLGALKG & KTV------- & 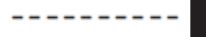 & FLVAATLRPE \\
\hline Mus & $----V--L E P$ & YPSKLSGLKG & KN I------ & 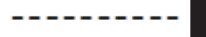 & FLVAATLRPE \\
\hline Xenopus_laevis & $----V--L E P$ & LPTKLSGLKG & RNV ------- & ---------- & FLVAATLRPE \\
\hline \multirow[t]{2}{*}{ Consistency } & 0000800775 & 5846764766 & 7480000000 & 0000000000 & $78898 * 8 * * 9$ \\
\hline & $\ldots \ldots 360$ & .370 & $\ldots \ldots \ldots 380$ & . . 39 & \\
\hline $\operatorname{col}$ & 1 TFMGCTYLAV & AAGHPLAQK- & $-----A A E N N$ & PELAAFIDEC & RNTKVAEAE- \\
\hline s_c & CTMYGQTCCFV & SPTIEYGIFD & $A G--D S Y F I T$ & TERAFKNMSY & KRGF \\
\hline tha & aTMYGQTNAWV & L PDGKYGAYE & IS-ETEVFIL & TERAALNLAY & NPQEP \\
\hline Dro & A TMYGQTNCWL & H PDIKY I AWQ & ANKNNEVWVS & TRRAARNMTY & VEGEI \\
\hline Ano & TMFGQTNCWV & RPDMKYIGFE & TL-NGDIFIC & TQRAARNMSY & I NGVV \\
\hline _gut & TMFGQTNCWL & RPDMKYIGFE & TG-GGDIFIC & SQRAARNMSY & QGFTRDNGVL \\
\hline Homo & TMFGQTNCWV & RPDMKYIGFE & TV-NGDIFIC & TQKA ARNMSY & DNGVV \\
\hline Dan & TMFGQTNCW I & RPDMKYVVFE & TA-NGDLFIS & TQRSARNMSY & QGFTKENGVV \\
\hline Mus & TMFGQTNCWV & RPDMKYIGFE & TA-NGDIFIC & TQRAARNMSY & NGVV \\
\hline Xenopus_laevis & TMFGQTNCWL & RPDMPYIAFE & TT-NGDIFIC & TQRAARNMSY & NGVV \\
\hline \multirow[t]{2}{*}{ Consistency } & $\star 87 \star 8 \star 6778$ & 5875685466 & 5304466784 & 7779868778 & 9677535754 \\
\hline & 410 & $\ldots \ldots 420$ & .431 & & 450 \\
\hline $\operatorname{col}$ & 1 MATMEKKG-V & DTGFKAVHPL & TGEEIPVWAA & N- -FVLMEYG & TGAVMAVPGH \\
\hline s_c & CKPIVTVPGKA & FIGTKIHAPQ & SVYPE-LR I L & PMETVIATKG & TGVVTCVPSN \\
\hline tha & A SCLVETGYD & L I GL P LRSPL & SVNEI-IYAL & PMLTILTNKG & TGIVTSVPSD \\
\hline Drosophil & aKVLAEVTGQD & LLGVPLSAPL & TKHKV-VYSL & PMLSIKEDKG & TGVVTSVPSD \\
\hline Anolis_c & PPVKELMGED & ILGAPISAPL & TSYKV-IYTL & PMLTIKEDKG & TGVVTSVPSD \\
\hline Taeniopy & TPVKELMGEE & ILGAAISAPI & TNYKV-IYAL & PMLTIKEDKG & TGVVTSVPSD \\
\hline Homo_sapiens & PVVKELMGEE & I LGASLSAPL & TSYKV-IYVL & PMLTIKEDKG & TGVVTSVPSD \\
\hline Danio_rerio & PVIMNIMGQD & I LGCAISAPL & TSYKT-IYAL & PMLTIKEDKG & TGVVTSVPSD \\
\hline Mus_musculus & PVVKELMGEE & ILGASLSAPL & TCYKV-VYVL & PMLTIKEDKG & TGVVTSVPSD \\
\hline Xenopus_laevis & PVVKELMGED & LLGAALSAPL & TSYKV-IYAL & PMLTIKEDKG & TGVVTSVPSD \\
\hline \multirow[t]{2}{*}{ Consistency } & $5675775 * 55$ & $67 * 54867 * 8$ & $84677 \overline{08768}$ & 886796678 * & $\star \star 8 * 87 \star \star 87$ \\
\hline & $\ldots \ldots \ldots 460$ & $\ldots \ldots \ldots .470$ & $\ldots .480$ & $\ldots \ldots \ldots .490$ & $\ldots \ldots \ldots 500$ \\
\hline & DQRDYEFASK & 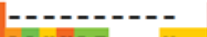 & YGI------ & - NIKPVIL- & - - - - - - \\
\hline & $=S$ PDDYITTKD & L L Н K P E $---Y$ & Y G IKPEWIDH & - E IVP IMHTE & KYGDLTAKAI \\
\hline Arabidopsis_tha & $A P D D Y M A L Q D$ & L IKKPALQDK & YGVKTEWLPT & -EIIPIINIP & EFGDKAAEKV \\
\hline ophila_mela: & SPDDYAALVD & LQKKEAFRQK & Y GLKDEMVLP & YEP I P I IEVP & TLGKLSAVHA \\
\hline Anolis_caroline & SPDDIAALRD & LKKKQPFRAK & Y GIKDDMVLP & FEPVPVIEIP & GYGQLSAPMI \\
\hline Taeniopygia_gut & $=S P D D F A A L R D$ & LKKKQALRVK & YGIRDEMVLP & FEPVPIIEIP & GYGSLCAPFV \\
\hline Homo_sapiens & SPDDIAALRD & L KKKQALRAK & Y GIRDDMVLP & FEPVPVIEIP & GFGNLSAVTI \\
\hline Danio_rerio & A P D D I A A L RD & IKKKQALREK & $Y G I Q D F M V L P$ & FEPVPIIEIP & GYGNISAP IV \\
\hline & SPDDLAALRD & L K K K Q A L R TK & F GIRDDMVLP & FEPV PVLEIP & G I GN L P AVTV \\
\hline & APDDIAALRD & LKKKQALRQK & YGIKDEMVLP & FEPVPIIDIP & GYGNLSAPAV \\
\hline Consistency & $788 * 567768$ & 7468554526 & $9 * 86555745$ & $4858 * 98566$ & 4584648326 \\
\hline
\end{tabular}

\section{Unconserved 1123456789 Conserved}

Figure 4. ${ }^{39}$ Multiple sequence alignment of the CP1 Editing Domain. The black box on the multiple sequence alignment highlights the "threonine-rich" region. The threonine-rich region provides chemical specificity to the CP1 domain hydrolytic active site. Organism are as follows: Escherichia coli, Saccharomyces cerevisiae, Arabidopsis thaliana, Draosopbila melanogaster, Danio rerio, Xenopus laevis, Anolis carolinensis, Taeniopygia guttata, Mus musculus, and Homo sapiens. 


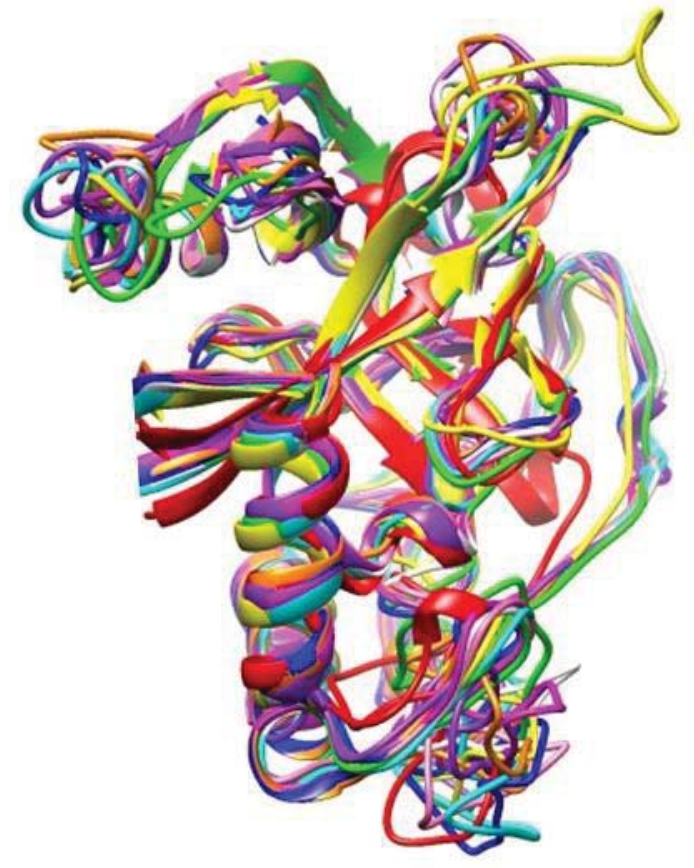

Figure 5A.

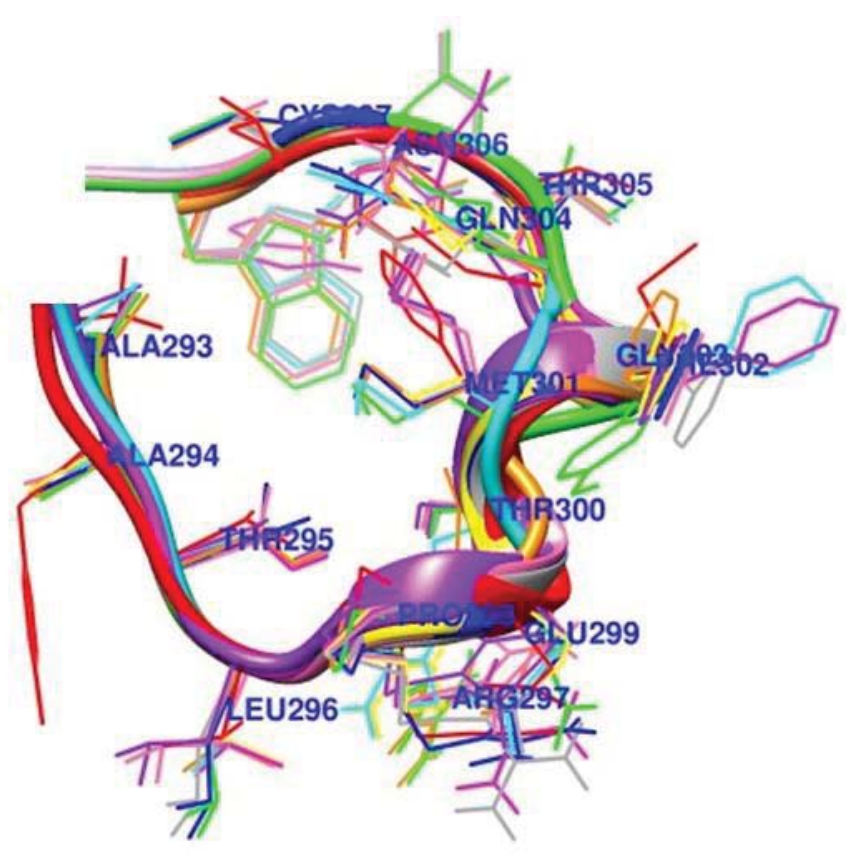

Figure 5B.

Figure 5. CP1 Domain superimposition. ${ }^{37,39}$ 5A. Superimposition of the entire CP1 domain. 5B. Superimposition of the "threonine-rich" region within the hydrolytic active site of the CP1 domain. The E. coli LeuRS X-ray crystallography structure is red. The E. coli LeuRS crystal structure is overlaid with the following computationally generated LeuRS homology models: S. cerevisiae (yellow), A. thaliana (purple), D. melanogaster (green), D. rerio (orange), X. laevis (blue), A. carolinensis (magenta), T. guttate (cyan), M. musculus (gray), and H. sapiens (pink).

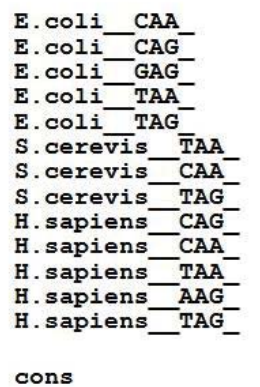

BAD AVG GOOD

GCCGAAGTGGCGAAATCGGTAGACGCAGTTGAT TCAAAATCAACCGTAG--AAATACGTGCCGGTT CGAGTCCGGCCTTCGGCACCA GCGAAGGTGGCGGAATTGGTAGACGCGCTAGCT TCAGGTGTTAGTGTCCTTACGGACGTGGGGGTT CAAGTCCCCCCCCTCGCACCA GCCGAGGTGGTGGAATTGGTAGACACGCTACCT TGAGGTGGTAGTGCCCAATAGGGCTTACGGGTT CAAGTCCCGTCCTCGGTACCA GCCCGGATGGTGGAATCGGTAGACACAAGGGAT TTAAAATCCCTCGGCGTTCGCGCTGTGCGGGTT CAAGTCCCGCTCCGGGTACCA GCGGGAGTGGCGAAATTGGTAGACGCACCAGAT TTAGGTTCTGGCGCC-GGAGGGTTGGCCGAGT-GGTA-AGGCGGCAGACTTAAGATCTGTTGGACGGTTGTCCGCGCGAGTT CGAACCTCGCA---TCCTTCA GGTTGTTTGGCCGAGC-GGTA-AGGCGCCTGAT TCAAGCTCAGGTAT-C-GTAAGATGCAAGAGTT CGAATCTCTTA---GCAACCA GGTTGTTTGGCCGAGC-GGTA-AGGCGCCTGAT TCAAGCTCAGGTAT-C-GTAAGATGCAAGAGTTCGAATCTCTTA---GCAACCA GTCAGGATGGCCGAGC-GGTA-AGGCGCTGCGT TCAGGTCGCAGTCTCCC-CTGGAGGCGTGGGTT CGAATCCCACT---CCTGACA GTCAGGATGGCCGAGT-GGTA-AGGCGCCAGAC TCAAGTTCTGGTCTCCGGATGGAGGCGTGGGTTCGAATCCCACT---TCTGACA GTTAAGATGGCAGAGC-CGCAATTGCATAAAAC TTAAAAC------T-T--TACAA--CAGAGGTT CAACTCCTCTT---CTTAACA GGGCCAGTGGCTCAAT-GGAA-ATGCGTCTGACTAAGAATCAG--------AAGAT--CCAGCCT TGACTCCTGGC---TGGCTCA GGTAGTGTGGTTGAAT-GGTA-AGGCACTGAATTTAGGCTCCAGTCT-C-TTTGGGGACGTGGGTT TAAATCCCACT---GCTGCAA

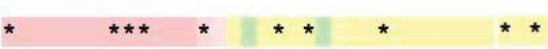

Figure 6. Multiple sequence alignment of tRNA Leu Isotypes. The multiple sequence alignment of tRNALeu from E. coli, S. cerevisiae, and H. sapiens shows sequence variability amongst these different isotopic forms. The tRNA Leu isotypes of CAA, CAG, GAG, TAA, TAG, and AAG originate from E. coli, S. cerevisiae, and H.

sapiens. These three organisms were chosen because they represent the greatest evolutionary time lapse amongst organisms on the eukaryotic branch.

LeuRS domain insertions are thought to be later evolutionary additions to LeuRS, to improve catalytic efficiency. ${ }^{16,21,42}$ Indeed, the auxiliary LeuRS domains like the CP1 domain and the ZN-1 domain have been biochemically analyzed and shown to drastically improve LeuRS catalysis. ${ }^{39,} 43$ The CP1 domain is a unique insertion into the Rossmann fold and serves to hydrolyze misaminoacylated products. ${ }^{39}$ The CP1 domain is crucial to maintain LeuRS function and fidelity due to the structurally promiscuous nature of the aminoacylation active site, which can readily accommodate structural homologs of leucine. ${ }^{44}$ The structural ambiguity of the aminoacylation active site would be a natural evolutionary consequence since LeuRS, IleRS and ValRS all structurally diverged from a common aaRS ancestor and the cognate amino acids that these aaRSs charge, namely leucine, isoleucine and valine, are all structurally similar. ${ }^{16,21,42}$ Therefore, the CP1 domain functions as an editing domain that allows the LeuRS enzyme to distinguish between leucine and other amino acid structural homologs. To determine the extent of conservation within the CP1 domain, sequence alignment data was gathered for the entire sequences of the CP1 domains from E. coli, $S$. 
cerevisiae, A. thaliana, D. melanogaster, D. rerio, X. laevis, A. carolinensis, T. guttata, M. musculus and H. sapiens (Fig. 4). From this data, it is evident that there is significant sequence conservation within the CP1 domains for these organisms, with the one outlier being E. coli. This finding may be a computational artifact due to E. coli's CP1 domain being generally smaller than the CP1 domains from the eukaryotic sources. Based on the sequence alignment, the CP1 domains from E. coli and S. cerevisiae had 31\% identity and 48\% homology. The CP1 domains from S. cerevisiae and $A$. thaliana share 45\% identity and 64\% homology. The CP1 domains from $A$. thaliana and D. melanogaster share $56 \%$ identity and $73 \%$ homology. The CP1 domains from D. melanogaster and D. rerio share 64\% identity and 81\% homology. The CP1 domains from D. rerio and X. laevis share $84 \%$ identity and $92 \%$ homology. The CP1 domains from $X$. laevis and $A$. carolinensis share $87 \%$ identity and 93\% homology. The CP1 domains from $A$. carolinensis and $T$. guttata share 88\% identity and 94\% homology. The CP1 domains from T. guttata and M. musculus share 85\% identity and 94\% homology. The CP1 domains from M. musculus and H. sapiens share 93\% identity and 94\% homology. Therefore, to build upon these results, structural superimpositions of the entire CP1 domain as well as the hydrolytic active site were generated (Fig. 5A and 5B). Computational models were generated for all CP1 domains to provide consistent analysis, which were based off existing crystal structures of LeuRS. From Figure 5A, there is considerable structural conservation within all the CP1 domain structures. Some structural variation is observed within the loop regions of the CP1 domain. This finding is expected since these disorganized protein structural features are difficult to computationally model. Therefore, these slight structural perturbations may be a computational artifact. An alternative explanation could be that some of these loop regions could interact with tRNALeu, helping to facilitate its translocation between the active sites of LeuRS. ${ }^{45}$ Since there is variability amongst tRNA ${ }^{\text {Leu }}$ sequences across species lines (Fig. 6), a reasonable assumption would be that regions within the CP1 domain that interact and bind with tRNA ${ }^{\text {Leu }}$ would also have variability.

Close examination of the CP1 domain hydrolytic active site pocket in Figure 5B indicates that there is considerable structural conservation. Indeed, amongst the eukaryotic species the hydrolytic active site sequences that comprise the threonine-rich region are completely conserved. ${ }^{39}$ When compared to the E. coli threonine-rich region, there are only two amino acids that deviate from the eukaryotic sequences, the T248 and D251 residues. A similar result was found by Pang. et. al. through an amino acid sequence comparison of the threonine-rich region from dissimilar organisms such as E. coli and H. sapiens. ${ }^{46}$ Evolutionarily, this would indicate that the CP1 domain would have evolved early-on as LeuRS structurally diverged from its common ancestral aaRS to provide chemical specificity for the accurate incorporation of leucine during protein synthesis. Thus, based on these results, we hypothesize that the CP1 domain would have probably been the first leucine-specific domain adaptation, which allowed a more catalytically efficient LeuRS to replace an existing ribozyme for leucine aminoacylation catalysis.

In addition to examining how the aminoacylation and CP1 domain active sites evolved, other sequence and structural features were studied to gain a more global perspective on how LeuRS evolved through the speciation of eukaryotes. Therefore, a qualitative evolutionary tree was generated to visually follow the molecular evolution of LeuRS structure through the speciation of eukaryotes starting from a bacterial example (Fig. 7). Each node represents a major split between taxa within the eukaryotic domain of life. ${ }^{40}$ Since it is thought that the eukaryotic domain of life is rooted from the bacterial domain, the E. coli LeuRS structure was used as the initial point of reference. ${ }^{22,41}$ Hence, the E. coli LeuRS structure is entirely gray, any subsequent changes within the LeuRS structure are highlighted in red. Structural deviations for each species were determined through generating a sequence alignment of the LeuRS from the organism of interest and the LeuRS from the organism on the next lower branch. These pairwise sequence alignments were generated using the PRALINE sequence alignment tool. Residues with a conservation score of "4" or less were highlighted in red. Therefore, the S. cerevisiae LeuRS structural deviation from E. coli LeuRS was determined through a sequence alignment of $E$. coli LeuRS and $S$. cerevisiae LeuRS. In like manner, the $A$. thaliana LeuRS structural deviation from $S$. cerevisiae LeuRS was determined through a sequence alignment of $S$. cerevisiae LeuRS and $A$. thaliana LeuRS. This same pattern of sequence comparison for each LeuRS was used to determine LeuRS structural deviations throughout the evolutionary tree. 


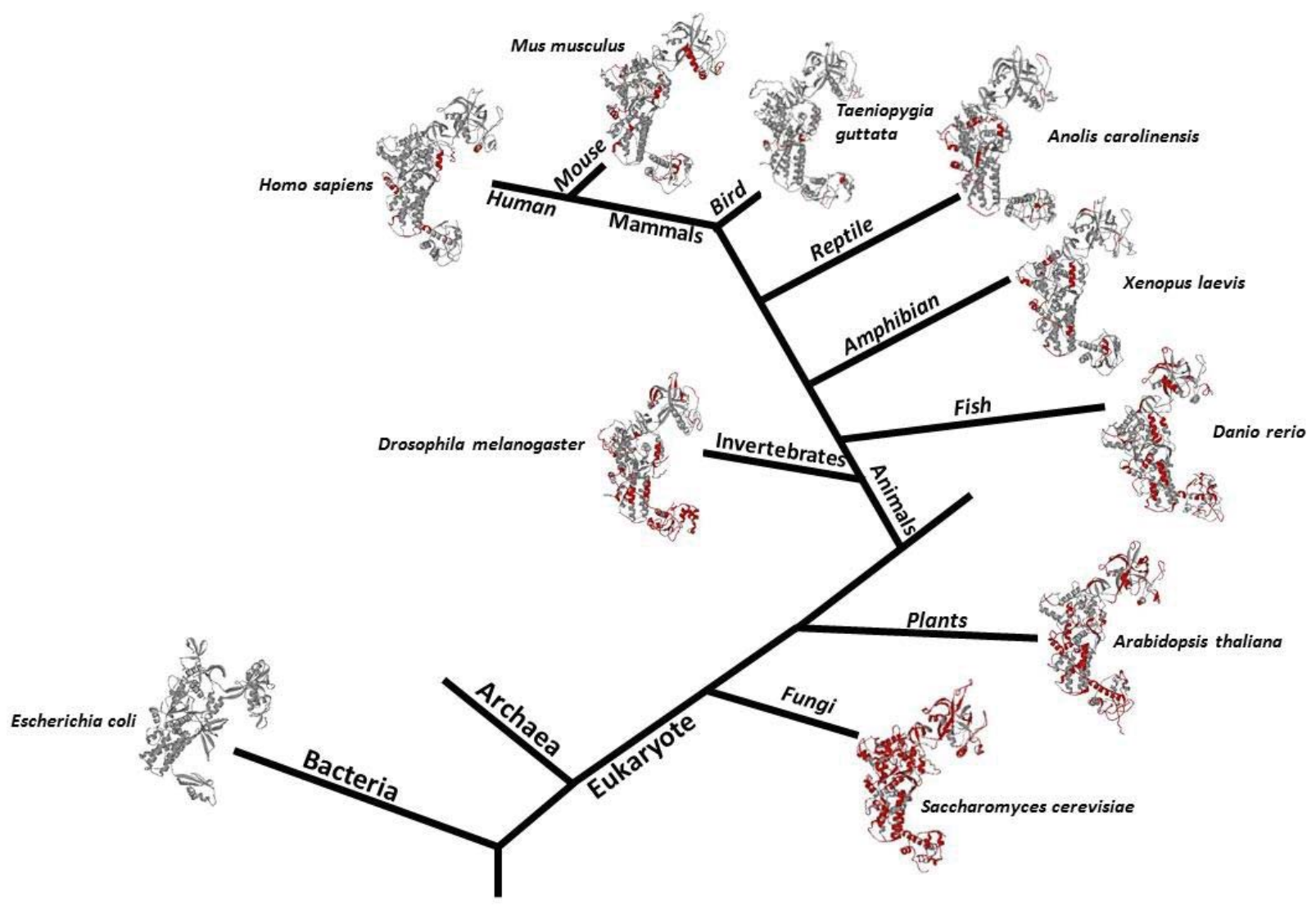

Figure 7. Evolutionary changes of LeuRS Among Eukaryotes. This qualitative phylogenetic tree compares LeuRS structures from each taxon within the eukaryotic domain of life, starting from a bacterial example. Universal features of LeuRS are gray and regions that have been subject to evolutionary change are red. Since the eukaryotic domain is thought to have arisen from bacterial origins, the E. coli LeuRS crystal structure is entirely gray ${ }^{22,37}$.

Figure 7 shows that there is significant structural deviation in the initial comparison between E. coli LeuRS and S. cerevisiae LeuRS. In part, this structural deviation is due to the size difference between these two enzymes. The S. cerevisiae LeuRS enzyme is 230 amino acids larger than the E. coli LeuRS enzyme. The size difference between these two enzymes as well as the other eukaryotic LeuRSs highlighted in this study could be due to additional non-translational catalytic functionality that was assumed by eukaryotic LeuRSs through the evolutionary process. ${ }^{47}$ Emerging evidence indicates that many aaRSs within eukaryotes have evolved critical roles that maintain cellular function outside of their canonical role in translation. ${ }^{47}$ Specifically, eukaryotic LeuRSs are known to participate in the TOR metabolic pathway, which functions to maintain amino acid metabolism. . $^{4-49}$

The observed sequence and structural deviations seem to occur within scaffolding residues. As has been previously stated, the aminoacylation active site and CP1 domain hydrolytic active site are highly conserved, thus structural deviation was not observed within these regions. The highest frequency of sequence and structural changes were observed between the lower-order eukaryotic species, namely; S. cerevisiae, A. thaliana, D. melanogaster and D. rerio. These sequence and structural differences may be due to mechanistic adaptation within the catalytic functionality of these enzymes. For example, it has been reported that $S$. cerevisiae LeuRS has an altered mechanistic behavior within the TOR metabolic pathway when compared to that of H. sapiens LeuRS mechanistic behavior within the same pathway. ${ }^{48-50}$ Mechanistic adaptation would be a natural consequence of the evolutionary process, especially since these enzymes function in different cellular environments with different extracellular ques. When considering the high frequency of sequence and structural changes within the lower-order eukaryotes, it is important to consider that this set of organisms differs greatly in their overall biology. The diversity within the lower-order eukaryotes can most simply be stated through general comparisons between the organisms, such as: single-cellular vs. multi-cellular; photosynthetic vs. non-photosynthetic; terrestrial vs. aquatic. Diversity within these biological systems would necessitate evolutionary adaptation, giving rise to LeuRS enzymes that have altered mechanistic function and potentially a different repertoire of molecular binding partners within each of these cell types. To further support this assertion, close examination of the LeuRS structures originating 
from higher-order organisms along the animal branch, namely; X. laevis, A. carolinensis, T. guttata, M. musculus, and H. sapiens, have very little sequence and structural deviation. Again, a general biological comparison of these organisms could be used to explain these results. This group of animals are multicellular, terrestrial and share many of the same physiological processes, albeit with some obvious differences. In short, these organisms are more biologically related than the lower-order eukaryotes. Within the higher-order eukaryotes, subgroup comparisons of LeuRSs shows extreme conservation. These subgroups are $A$. carolinensis : $T$. guttata and M. musculus: H. sapiens. This finding is not surprising given that lizards and birds are close evolutionary relatives. ${ }^{51}$ The evolutionary relationship between mice and humans is even more closely linked, as they are both mammals and are separated by only 96 million years of evolutionary time: less than half of the evolutionary time that separates lizards and birds. ${ }^{51-53}$

The evolutionary relationships between eukaryotic LeuRS enzymes that have been reported herein were further verified through the generation of a quantitative molecular phylogenetic tree based on LeuRS amino acid sequences (Fig. 8). This phylogenetic tree was developed through the PhyloT software program and verified through sequence analysis for the evolutionary hierarchy established with the Figure 7 results. The phylogenetic tree indicates that E. coli LeuRS and S. cerevisiae LeuRS are more closely related to each other when compared to the other lower-order eukaryotic organisms. From $A$. thaliana LeuRS to X. leavis LeuRS there is step-wise evolutionary relatedness for each of the branch points. This degree of evolutionary connectivity between the lower-order organisms has been previously explained for the Figure 7 results. In like manner, the subgroupings of $A$. carolinensis : T. guttata and M. musculus: H. sapiens LeuRS enzymes within this molecular phylogenetic tree have also been explained in the context of Figure 7 results. Therefore, the molecular phylogenetic tree confirms the Figure 7 results and associated hypotheses.

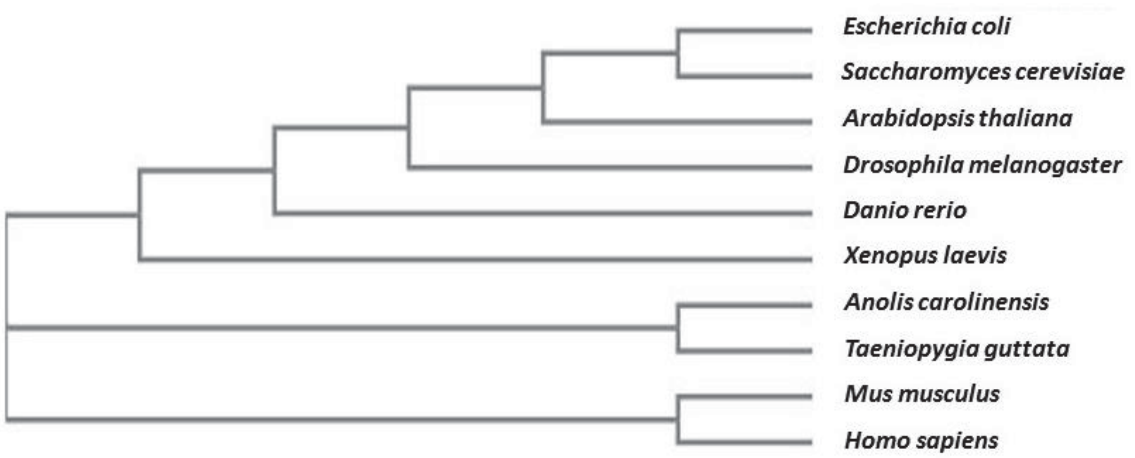

Figure 8. Molecular Phylogenetic Tree of Eukaryotic LeuRS. ${ }^{22}$ This phylogenetic tree compares LeuRS amino acid sequences from each taxon within the eukaryotic domain of life, starting from a bacterial example. Since the eukaryotic domain is thought to have arisen from bacterial origins, the E. coli LeuRS sequence is included.

Lastly, each of the LeuRS homology structures were superimposed on their nearest evolutionary relative using the Chimera software program. Therefore, E. coli LeuRS and S. cerevisiae LeuRS were superimposed on one another (Fig. 9A). This superimposition provided an essential structural comparison of these two LeuRS enzymes. Again, E. coli LeuRS is in red and $S$. cerevisiae LeuRS is in yellow. From this structural overlap, the greatest differences are within the CP1 and C-terminal domains. These differences are largely related to the overall size of these domains. Given that $S$. cerevisiae LeuRS has adopted additional non-translational functionality, these size differences could support these non-translational catalytic and binding functions. hese LeuRS structural differences may be due to sequence differences within the individual tRNA ${ }^{\text {Leu }}$ for each organism, which subsequently affects the tRNA ${ }^{\mathrm{Leu}}$ structures (Fig. 6).

The superimposition of $A$. thaliana LeuRS (purple) and D. melanogaster LeuRS (green) (Fig. 9B) as well as the superimposition of D. rerio LeuRS (orange) and X. laevis LeuRS (blue) (Fig. 9C) shows some structural differences largely within the CP1 and Cterminal domains. Again, these differences may be due to the very different biological environments in which these LeuRS enzymes operate, which would give rise to mechanistic differences in the catalytic functions of these enzymes. The superimposition of $A$. carolinensis, LeuRS (magenta) and T. guttate LeuRS (cyan) indicates that these enzymes are almost identical, albeit with some slight changes in a secondary structural elemental feature within the aminoacylation domain for $T$. guttate LeuRS, which may be an artifact of the protein modeling process (Fig. 9D). The fact that these two enzymes are structurally very similar is indicative of their close evolutionary relatedness, as previously explained. Lastly, the superimposition of the M. musculus LeuRS (gray) and H. sapiens LeuRS (pink) enzymes shows that they are virtually identical (Fig. 9E). This was an expected result given that 
these enzymes have mammalian origins, which are separated by very little evolutionary time. Thus, the superimposition of these LeuRS structures spanning the eukaryotic lineage supports our previous evolutionary assertions as well as provides the data necessary to visually compare how LeuRS changed or was conserved through evolutionary time.

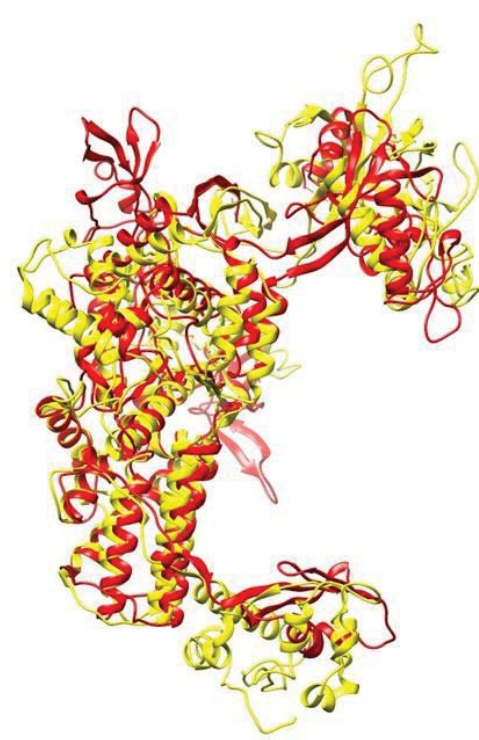

Figure 9A.

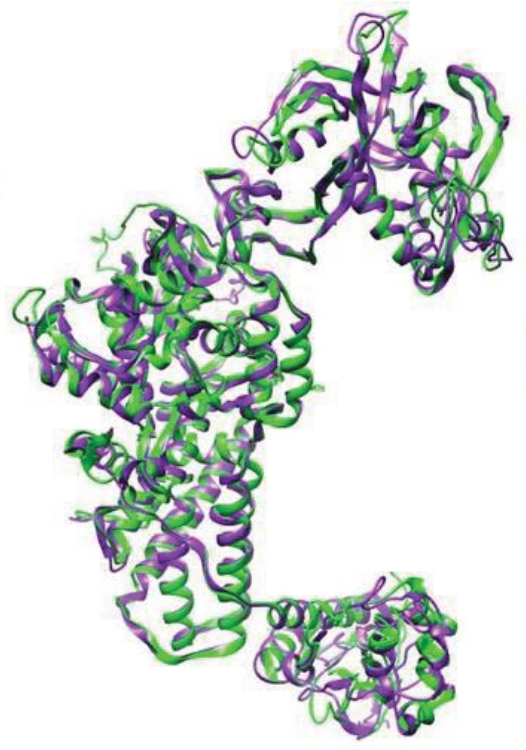

Figure 9B.

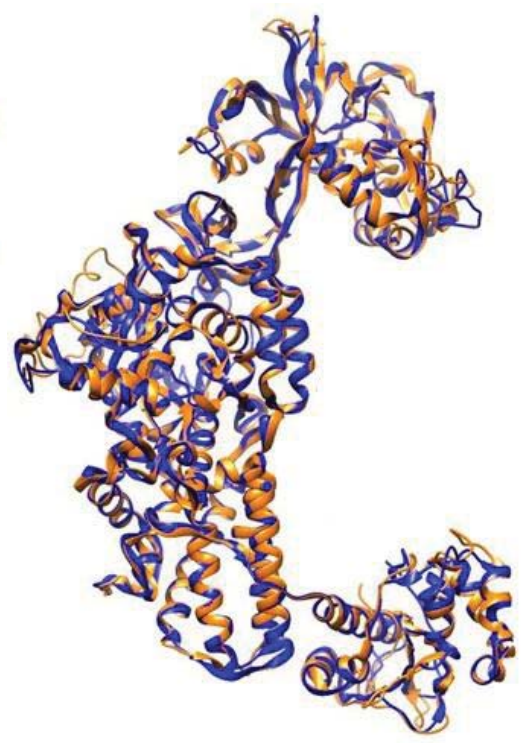

Figure 9C.

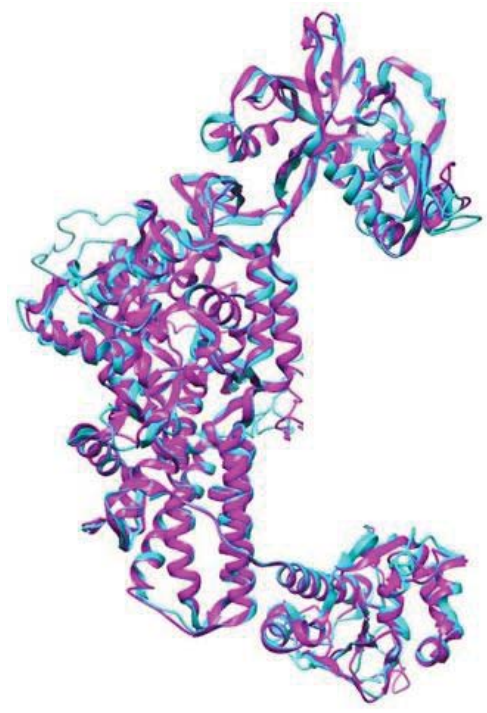

Figure 9D.

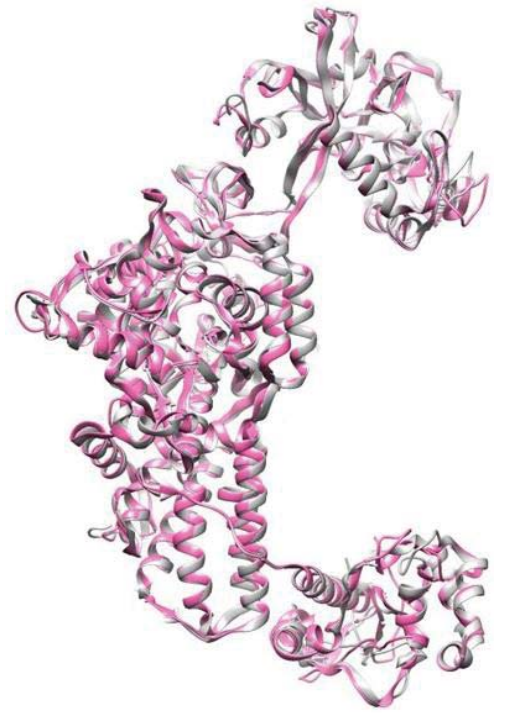

Figure 9E.

Figure 9. ${ }^{37}$ Superimposition of the LeuRS enzyme structures to compare and contrast structural changes amongst eukaryotic organisms.

9A. The E. coli LeuRS X-ray crystallography structure is red, overlaid with the $S$. cerevisiae LeuRS homology model in yellow.

9B. The A. thaliana LeuRS homology model (purple) is overlaid with the D. melanogaster LeuRS homology model (green). 9C. The D. rerio LeuRS homology model (orange) is overlaid with the X. laevis LeuRS homology model (blue).

9D. The A. carolinensis LeuRS homology model (magenta) is overlaid with the T. guttate LeuRS homology model (cyan). 9E. The M. musculus LeuRS homology model (gray) is overlaid with the H. sapiens LeuRS homology model (pink). 


\section{DISCUSSION}

The evolutionary origins of aaRSs have been well characterized by numerous research studies focused on understanding the development of the aminoacylation domain and aaRS catalytic specificity. 1, 5, 7-9, 21 Consensus among these research efforts is that aaRS enzymes have ancient origins that predate LUCA. To build upon these previously reported findings, this research effort aimed to understand the more contemporary evolutionary history of LeuRS, specifically through the eukaryotic lineage. The results reported herein indicate that the aminoacylation active site as well as the CP1 domain active site are both highly conserved. Indeed, a comparison of these active sites across domain and species lines indicates that there is very little variation within the sequences or structures of LeuRSs from these different species. This was a surprising result given that there are billions of years of evolutionary history separating the species highlighted in this research study.

Though the active sites of LeuRS are highly conserved, the results indicated that there was sequential and structural variation that occurred mainly within the scaffold residues. Particularly scaffold residues within the CP1 and C-terminal domains. A dramatic example of LeuRS structural variation were the superimposed structures of E. coli LeuRS and S. cerevisiae LeuRS enzymes, which was not surprising given that these enzymes originate from organisms in different domains of life and are separated by approximately 1.5 billion years of evolutionary time. ${ }^{14,40}$ Accordingly, these enzymes had the most sequence and structural variation compared with the other enzymes in this research study, which is likely due to non-translational functionality assumed by LeuRS enzymes through eukaryotic speciation to facilitate complex biological processes not present in bacteria. However, with this noted, the active sites for these enzymes were highly conserved, supporting the hypothesis that LeuRS enzymes diverged from a common ancestor aaRS., 9

Additional sequential and structural comparisons of LeuRS enzymes were generated, determining that through the speciation of eukaryotes there were only minor evolutionary changes. This was especially apparent when comparing LeuRS enzymes amongst higher-order eukaryotes. Comparisons between A. carolinensis LeuRS : T. guttate LeuRS and M. musculus LeuRS : H. sapiens LeuRS indicated very minor structural deviations. This result could be due to the relative short amount of evolutionary time between these organisms as well as their close physiologies. ${ }^{51-53}$ Contrasting this result with the lower-order organisms, the LeuRS enzymes within this group displayed more variability. This is likely due to longer evolutionary time spans between these organisms as well as dissimilar physiologies. ${ }^{40}$ Thus, the eukaryotic lineage carefully maintained LeuRS structure through the speciation process with the actives sites being strictly conserved.

\section{CONCLUSIONS}

These research findings show a high level of sequence and structural conservation amongst the eukaryotic LeuRS enzymes that were examined. Evolutionarily, this would indicate that vertical gene transfer events fostered the propagation of LeuRS through the speciation of eukaryotes. Unlike what has been reported for the bacterial and Archaeal domains, which have both been shown to have experienced LeuRS HGT and domain acquisition events. ${ }^{7-9}$ Additionally, both bacterial and Archaeal domains have robust mechanisms in place to facilitate HGT events. Thus, these results are expected, especially since the Eukaryota domain was the last domain to have emerged through the evolution of life and is more molecularly complex. ${ }^{40}$ Therefore, gene transfer events in eukaryotic cells are more restrictive than gene transfer events in bacteria or Archaea, thus the likelihood of LeuRS HGT events occurring amongst eukaryotes is almost non-existent. Especially due to LeuRS being part of the translational machinery. Enzymes that mechanistically drive the molecular central dogma were likely acquired before or at the time of LUCA, thus many of them would phylogenetically be deeply rooted at the base of the tree of life. Therefore, later acquisition of these enzymes by eukaryotes is improbable. As such, we propose that LeuRS was transferred through the eukaryotic speciation process via vertical gene transfer, which explains the high rate of LeuRS conservation within each taxa of the eukaryotic domain.

\section{ACKNOWLEDGEMENTS}

The authors thank the University of Nebraska system for supporting this research effort. 


\section{REFERENCES}

1. O'Donoghue, P.; Luthey-Schulten, Z., (2003) On the evolution of structure in aminoacyl-tRNA synthetases. Microbiol Mol Biol Rev, 67 (4), 550-573.

2. Lee, N.; Bessho, Y.; Wei, K.; Szostak, J. W.; Suga, H., (2000) Ribozyme-catalyzed tRNA aminoacylation. Nat Struct Biol, 7 (1), 28-33.

3. Arnez, J. G.; Moras, D., (1997) Structural and functional considerations of the aminoacylation reaction. Trends Biochem Sci, $22(6), 211-216$.

4. Ofengand, E. J.; Dieckmann, M.; Berg, P., (1961) The enzymic synthesis of amino acyl derivatives of ribonucleic acid. III. Isolation of amino acid-acceptor ribonucleic acids from Escherichia coli. J Biol Chem, 236, 1741-1747.

5. Woese, C. R.; Olsen, G. J.; Ibba, M.; Soll, D., (2000) Aminoacyl-tRNA synthetases, the genetic code, and the evolutionary process. Microbiol Mol Biol Rev, 64 (1), 202-236.

6. Wolf, Y. I.; Aravind, L.; Grishin, N. V.; Koonin, E. V., (1999) Evolution of aminoacyl-tRNA synthetases--analysis of unique domain architectures and phylogenetic trees reveals a complex history of horizontal gene transfer events. Genome Res, 9 (8), 689-710.

7. Fournier, G. P.; Andam, C. P.; Alm, E. J.; Gogarten, J. P., (2011) Molecular evolution of aminoacyl tRNA synthetase proteins in the early history of life. Orig Life Evol Biosph, 41 (6), 621-632.

8. Andam, C. P.; Harlow, T. J.; Papke, R. T.; Gogarten, J. P., (2012) Ancient origin of the divergent forms of leucyl-tRNA synthetases in the Halobacteriales. BMC Evol Biol, 12, 85.

9. Dohm, J. C.; Vingron, M.; Staub, E., (2006) Horizontal gene transfer in aminoacyl-tRNA synthetases including leucine-specific subtypes. J Mol Evol, 63 (4), 437-447.

10. Eriani, G.; Delarue, M.; Poch, O.; Gangloff, J.; Moras, D., (1990) Partition of tRNA synthetases into two classes based on mutually exclusive sets of sequence motifs. Nature, 347 (6289), 203-206.

11. Ibba, M.; Curnow, A. W.; Soll, D., (1997) Aminoacyl-tRNA synthesis: divergent routes to a common goal. Trends Biochem Sci, $22(2), 39-42$.

12. Burbaum, J. J.; Schimmel, P., (1991) Assembly of a class I tRNA synthetase from products of an artificially split gene. Biochemistry, 30 (2), 319-324.

13. Cusack, S., (1997) Aminoacyl-tRNA synthetases. Curr Opin Struct Biol, 7 (6), 881-889.

14. Woese, C. R.; Kandler, O.; Wheelis, M. L., (1990) Towards a natural system of organisms: proposal for the domains Archaea, Bacteria, and Eucarya. Proc Natl Acad Sci U S A, 87 (12), 4576-4579.

15. Brown, J. R.; Doolittle, W. F., (1995) Root of the universal tree of life based on ancient aminoacyl-tRNA synthetase gene duplications. Proc Natl Acad Sci U S A, 92 (7), 2441-2445.

16. Schimmel, P.; Ribas de Pouplana, L., (1995) Transfer RNA: from minihelix to genetic code. Cell, 81 (7), $983-986$.

17. Schmitt, E.; Meinnel, T.; Blanquet, S.; Mechulam, Y., (1994) Methionyl-tRNA synthetase needs an intact and mobile 332KMSKS336 motif in catalysis of methionyl adenylate formation. J Mol Biol, 242 (4), 566-576.

18. Webster, T.; Tsai, H.; Kula, M.; Mackie, G. A.; Schimmel, P., (1984) Specific sequence homology and three-dimensional structure of an aminoacyl transfer RNA synthetase. Science, 226 (4680), 1315-1317.

19. Cusack, S.; Yaremchuk, A.; Tukalo, M., (2000) The 2 A crystal structure of leucyl-tRNA synthetase and its complex with a leucyl-adenylate analogue. EMBO J, 19 (10), 2351-2361.

20. Eriani, G.; Cavarelli, J.; Martin, F.; Ador, L.; Rees, B.; Thierry, J. C.; Gangloff, J.; Moras, D., (1995) The class II aminoacyltRNA synthetases and their active site: evolutionary conservation of an ATP binding site. J Mol Evol, 40 (5), $499-508$.

21. Zhao, M. W.; Zhu, B.; Hao, R.; Xu, M. G.; Eriani, G.; Wang, E. D., (2005) Leucyl-tRNA synthetase from the ancestral bacterium Aquifex aeolicus contains relics of synthetase evolution. EMBO J, 24 (7), 1430-1439.

22. Iwabe, N.; Kuma, K.; Hasegawa, M.; Osawa, S.; Miyata, T., (1989) Evolutionary relationship of archaebacteria, eubacteria, and eukaryotes inferred from phylogenetic trees of duplicated genes. Proc Natl Acad Sci U S A, 86 (23), 9355-9359.

23. Altschul, S. F.; Gish, W.; Miller, W.; Myers, E. W.; Lipman, D. J., (1990) Basic local alignment search tool. J Mol Biol, 215 (3), 403-410.

24. Sievers, F.; Wilm, A.; Dineen, D.; Gibson, T. J.; Karplus, K.; Li, W.; Lopez, R.; McWilliam, H.; Remmert, M.; Soding, J.; Thompson, J. D.; Higgins, D. G., (2011) Fast, scalable generation of high-quality protein multiple sequence alignments using Clustal Omega. Mol Syst Biol, 7, 539.

25. Simossis, V. A.; Heringa, J., (2005) PRALINE: a multiple sequence alignment toolbox that integrates homology-extended and secondary structure information. Nucleic Acids Res, 33 (Web Server issue), W289-294.

26. Chan, P. P.; Lowe, T. M., (2009) GtRNAdb: a database of transfer RNA genes detected in genomic sequence. Nucleic Acids Res, 37 (Database issue), D93-97.

27. Di Tommaso, P.; Moretti, S.; Xenarios, I.; Orobitg, M.; Montanyola, A.; Chang, J. M.; Taly, J. F.; Notredame, C., (2011) TCoffee: a web server for the multiple sequence alignment of protein and RNA sequences using structural information and homology extension. Nucleic Acids Res, 39 (Web Server issue), W13-17.

28. Yang, Y.; Faraggi, E.; Zhao, H.; Zhou, Y., (2011) Improving protein fold recognition and template-based modeling by 
employing probabilistic-based matching between predicted one-dimensional structural properties of query and corresponding native properties of templates. Bioinformatics, 27 (15), 2076-2082.

29. Yang, J.; Zhang, Y., (2015) I-TASSER server: new development for protein structure and function predictions. Nucleic Acids Res, 43 (W1), W174-181.

30. Yang, J.; Yan, R.; Roy, A.; Xu, D.; Poisson, J.; Zhang, Y., (2015) The I-TASSER Suite: protein structure and function prediction. Nat Methods, 12 (1), 7-8.

31. Pettersen, E. F.; Goddard, T. D.; Huang, C. C.; Couch, G. S.; Greenblatt, D. M.; Meng, E. C.; Ferrin, T. E., (2004) UCSF Chimera--a visualization system for exploratory research and analysis. J Comput Chem, 25 (13), 1605-1612.

32. Sanner, M. F.; Olson, A. J.; Spehner, J. C., (1996) Reduced surface: an efficient way to compute molecular surfaces. Biopolymers, 38 (3), 305-320.

33. Harris, M.; Jones, T. A., (2001) Molray--a web interface between O and the POV-Ray ray tracer. Acta Crystallogr D Biol Crystallogr, 57 (Pt 8), 1201-1203.

34. Letunic, I.; Bork, P., (2007) Interactive Tree Of Life (iTOL): an online tool for phylogenetic tree display and annotation. Bioinformatics, 23 (1), 127-128.

35. Letunic, I.; Bork, P., (2016) Interactive tree of life (iTOL) v3: an online tool for the display and annotation of phylogenetic and other trees. Nucleic Acids Res, 44 (W1), W242-245.

36. Schimmel, P., (2008) Development of tRNA synthetases and connection to genetic code and disease. Protein Sci, 17 (10), 1643-1652.

37. Palencia, A.; Crepin, T.; Vu, M. T.; Lincecum, T. L., Jr.; Martinis, S. A.; Cusack, S., (2012) Structural dynamics of the aminoacylation and proofreading functional cycle of bacterial leucyl-tRNA synthetase. Nat Struct Mol Biol, 19 (7), $677-684$.

38. Schimmel, P. R.; Soll, D., (1979) Aminoacyl-tRNA synthetases: general features and recognition of transfer RNAs. Annu Rev Biochem, 48, 601-648.

39. Mursinna, R. S.; Lincecum, T. L., Jr.; Martinis, S. A., (2001) A conserved threonine within Escherichia coli leucyl-tRNA synthetase prevents hydrolytic editing of leucyl-tRNALeu. Biochemistry, 40 (18), 5376-5381.

40. Moreira, D.; Lopez-Garcia, P., (2015) Evolution of viruses and cells: do we need a fourth domain of life to explain the origin of eukaryotes? Philos Trans R Soc Lond B Biol Sci, 370 (1678), 20140327.

41. Gogarten, J. P.; Kibak, H.; Dittrich, P.; Taiz, L.; Bowman, E. J.; Bowman, B. J.; Manolson, M. F.; Poole, R. J.; Date, T.; Oshima, T.; et al., (1989) Evolution of the vacuolar H+-ATPase: implications for the origin of eukaryotes. Proc Natl Acad Sci U S A, 86 (17), 6661-6665.

42. Schimmel, P.; Ribas de Pouplana, L., (2001) Formation of two classes of tRNA synthetases in relation to editing functions and genetic code. Cold Spring Harb Symp Quant Biol, 66, 161-166.

43. Kumar, M.; Kumar, S. A.; Dimkovikj, A.; Baykal, L. N.; Banton, M. J.; Outlaw, M. M.; Polivka, K. E.; Hellmann-Whitaker, R. A., (2015) Zinc is the molecular "switch" that controls the catalytic cycle of bacterial leucyl-tRNA synthetase. J Inorg Biochem, 142, 59-67.

44. Fersht, A. R., (1998) Sieves in sequence. Science, 280 (5363), 541.

45. Hellmann, R. A.; Martinis, S. A., (2009) Defects in transient tRNA translocation bypass tRNA synthetase quality control mechanisms. J Biol Chem, 284 (17), 11478-11484.

46. Pang, Y. L.; Martinis, S. A., (2009) A paradigm shift for the amino acid editing mechanism of human cytoplasmic leucyl-tRNA synthetase. Biochemistry, 48 (38), 8958-8964.

47. Guo, M.; Schimmel, P., (2013) Essential nontranslational functions of tRNA synthetases. Nat Chem Biol, 9 (3), $145-153$.

48. Bonfils, G.; Jaquenoud, M.; Bontron, S.; Ostrowicz, C.; Ungermann, C.; De Virgilio, C., (2012) Leucyl-tRNA synthetase controls TORC1 via the EGO complex. Mol Cell, 46 (1), 105-110.

49. Han, J. M.; Jeong, S. J.; Park, M. C.; Kim, G.; Kwon, N. H.; Kim, H. K.; Ha, S. H.; Ryu, S. H.; Kim, S., (2012) Leucyl-tRNA synthetase is an intracellular leucine sensor for the mTORC1-signaling pathway. Cell, 149 (2), 410-424.

50. Duran, R. V.; Hall, M. N., (2012) Leucyl-tRNA synthetase: double duty in amino acid sensing. Cell Res, 22 (8), $1207-1209$.

51. Padian, K.; Chiappe, L. M., (1998) The origin of birds and their flight. Sci Am, 278 (2), 38-47.

52. Nei, M.; Xu, P.; Glazko, G., (2001) Estimation of divergence times from multiprotein sequences for a few mammalian species and several distantly related organisms. Proc Natl Acad Sci U S A, 98 (5), 2497-2502.

53. Green, R. E.; Braun, E. L.; Armstrong, J.; Earl, D.; Nguyen, N.; Hickey, G.; Vandewege, M. W.; St John, J. A.; CapellaGutierrez, S.; Castoe, T. A.; Kern, C.; Fujita, M. K.; Opazo, J. C.; Jurka, J.; Kojima, K. K.; Caballero, J.; Hubley, R. M.; Smit, A. F.; Platt, R. N.; Lavoie, C. A.; Ramakodi, M. P.; Finger, J. W., Jr.; Suh, A.; Isberg, S. R.; Miles, L.; Chong, A. Y.; Jaratlerdsiri, W.; Gongora, J.; Moran, C.; Iriarte, A.; McCormack, J.; Burgess, S. C.; Edwards, S. V.; Lyons, E.; Williams, C.; Breen, M.; Howard, J. T.; Gresham, C. R.; Peterson, D. G.; Schmitz, J.; Pollock, D. D.; Haussler, D.; Triplett, E. W.; Zhang, G.; Irie, N.; Jarvis, E. D.; Brochu, C. A.; Schmidt, C. J.; McCarthy, F. M.; Faircloth, B. C.; Hoffmann, F. G.; Glenn, T. C.; Gabaldon, T.; Paten, B.; Ray, D. A., (2014) Three crocodilian genomes reveal ancestral patterns of evolution among archosaurs. Science, 346 (6215), 1254449. 


\section{ABOUT THE STUDENT AUTHORS}

Katelin Arndt is currently attending the University of Nebraska, Kearney with a Comprehensive Biology major with an emphasis in Health Science. She is a part of the honors program and a member of Phi Eta Sigma. She is planning a career as a physician assistant. Katelin started undergraduate research under Doctor Rachel Whitaker in the fall of 2016 and hopes to continue research both throughout her academic career and as a medical professional.

Katelyn Unvert is a sophomore majoring in biology at the University of Nebraska, Kearney. She has previously published her research in the Journal of Inorganic Biochemistry and has presented this research at a national conference. She plans to continue to participate in the scientific process by conducting undergraduate research. Her future plans are to attend pharmacy school and conduct pharmacological research.

\section{PRESS SUMMARY}

Enzymes known as Aminoacly-tRNA synthetases are an important part of every known cell. Because they are so widespread, there is considerable research that can be done on how this enzyme has evolved over time. This study focuses on the specific enzyme leucyl-tRNA synthetase. It shows how the enzyme has changed, what aspects of it are essential to its function, and that it may have potential in pharmaceuticals. 